\title{
Transepithelial accelerated versus conventional corneal collagen crosslinking in patients with keratoconus: a comparative study
}

This article was published in the following Dove Medical Press journal: Clinical Ophthalmology

\author{
Carolina Madeira' \\ Ana Vasques ${ }^{2}$ \\ João Beato' \\ Gonçalo Godinho' \\ Luís Torrão' \\ Manuel Falcão ${ }^{1,3}$ \\ Fernando Falcão-Reis ${ }^{1,3}$ \\ João Pinheiro-Costa ${ }^{1,4}$ \\ 'Department of Ophthalmology, \\ Centro Hospitalar de São João, \\ Porto, Portugal; ${ }^{2}$ Faculty of Medicine, \\ University of Porto, Porto, Portugal; \\ ${ }^{3}$ Department of Surgery and \\ Physiology, Faculty of Medicine, \\ University of Porto, Porto, Portugal; \\ ${ }^{4}$ Department of Biomedicine, Faculty \\ of Medicine, University of Porto, \\ Porto, Portugal
}

Purpose: To systematically compare the efficacy of transepithelial accelerated corneal collagen crosslinking (TE-ACXL) with conventional corneal collagen crosslinking (C-CXL) in patients with progressive keratoconus.

Methods: Eyes of patients with progressive keratoconus who were treated with C-CXL $\left(3 \mathrm{~mW} / \mathrm{cm}^{2}\right.$ for 30 minutes) were compared with those who underwent TE-ACXL $\left(6 \mathrm{~mW} / \mathrm{cm}^{2}\right.$ for 15 minutes). Best-corrected visual acuity (BCVA), keratometry values, corneal thickness, and topometric indexes were compared before CXL, and at 2 months, 6 months, and 12 months postoperatively.

Results: The study enrolled 26 eyes of which 16 had TE-ACXL and 10 had C-CXL. Both groups were comparable at baseline and 12 months in terms of BCVA $(P=0.16$ and $P=0.57)$, Kmax (maximum keratometry) $(P=0.31$ and $P=0.73$ ), pachymetry $(P=0.75$ and $P=0.37)$, index of surface variance (ISV) $(P=0.45$ and $P=0.86)$, index of vertical asymmetry (IVA) $(P=0.26$ and $P=0.61$ ), and index of height decentration (IHD) $(P=0.27$ and $P=0.86$, respectively). We did not observe significant differences between preoperative and 12-month postoperative readings in within-group analysis: $\Delta \mathrm{Kmax}$ (TE-ACXL, $-2.13 \pm 5.41, P=0.25$ vs C-CXL, $0.78 \pm 1.65, P=0.17)$, $\Delta$ pachymetry (TE-ACXL, 4.10 $\pm 14.83, P=0.41$ vs C-CXL, $-8.90 \pm 22.09$, $P=0.24), \Delta \mathrm{ISV}(\mathrm{TE}-\mathrm{ACXL},-8.50 \pm 21.26, P=0.24$ vs $\mathrm{C}-\mathrm{CXL}, 3.80 \pm 12.43, P=0.36$ ), $\triangle$ IVA (TE-ACXL, $-0.12 \pm 0.31, P=0.26$ vs C-CXL, $0.03 \pm 0.18, P=0.61$ ), and $\Delta \mathrm{IHD}$ (TEACXL, $-0.03 \pm 0.07, P=0.18$ vs C-CXL, $-0.01 \pm 0.03, P=0.88)$.

Conclusion: Both TE-ACXL and C-CXL were similarly effective. Further follow-up is required to determine whether these techniques are comparable in the long-term.

Keywords: cornea, keratoconus, crosslinking, transepithelial, riboflavin, ultraviolet radiation, keratometry

\section{Introduction}

Keratoconus is an asymmetric, bilateral, and progressive corneal ectasia that can lead to visual impairment. ${ }^{1,2}$ It is the most common cause of primary keratectasia, with a prevalence in the general population as high as $1: 375 .{ }^{3,4}$ In keratoconic eyes, the cornea becomes thin and protrudes. ${ }^{2}$ It is believed that stromal thinning is due to collagen degradation by proteolytic enzymes or by decreased levels of proteinase inhibitors. ${ }^{1,5}$

Keratoconus management has changed. Treatment mainly included strategies to improve visual acuity (eg, rigid contact lens, corneal rings, and penetrating keratoplasty), and none of them could alter the natural history of the disease.$^{6-8}$ Corneal collagen crosslinking $(\mathrm{CXL})$ changed the perspective of keratoconus management because this treatment is able to slow or stop keratoconus progression. ${ }^{5,9,10}$ Since Wollensak et al
Correspondence: Carolina Madeira Department of Ophthalmology, Centro Hospitalar de São João, Avenida Prof Hernâni Monteiro, 4202 - 45I Porto, Portugal

Tel +35 I 2255 I 2100

Fax $+35|2255| 3669$

Email tania.carolina.madeira@gmail.com 
published the first report of CXL in 2003, many studies have been published that showed the efficacy and safety of conventional corneal collagen CXL (C-CXL). ${ }^{11-16}$

CXL consists of soaking the corneal stroma with riboflavin and then irradiating it with ultraviolet A (UVA) radiation. ${ }^{2,17}$ Even though the exact mechanism of action is still not completely clear, it is thought that the interaction between riboflavin and UVA results in the formation of oxygen free radicals that increase intra- and interfibrillar covalent bonds between the collagen fibrils. ${ }^{16,18,19}$

C-CXL protocols are based on the Dresden protocol. All the conventional protocols deliver 30 minutes of UVA radiation at an irradiance of $3.0 \mathrm{~mW} / \mathrm{cm}^{2}$, enabling a fluence of $5.4 \mathrm{~J} / \mathrm{cm}^{2}$, after the corneal instillation of riboflavin for 30 minutes. ${ }^{11,19,20}$

In an attempt to overcome the long duration of the conventional procedure, accelerated protocols were tested according to Bunsen-Roscoe's law of reciprocity that states that it is theoretically possible to achieve the same cumulative energy with an increased intensity of UVA radiation and shorter exposure. ${ }^{15,21}$

Epithelial debridement is performed to facilitate stromal riboflavin absorption, as riboflavin alone cannot pass through the intact epithelial cell membranes. ${ }^{22}$ However, in an attempt to reduce the risks of the epithelial removal (postoperative infection, pain and delay in visual rehabilitation, corneal scarring), transepithelial protocol has been tested. ${ }^{11,22,23}$

This protocol allows to overcome some limitations of conventional protocol, making it a more appealing and promising treatment option. However, medical literature on safety and efficacy of transepithelial accelerated corneal collagen crosslinking (TE-ACXL) remains scarce. ${ }^{24,25}$ Considering its advantages more studies to evaluate the role of this protocol in keratoconus treatment are needed.

The purpose of this study is to compare the efficacy of TE-ACXL with C-CXL in patients with progressive keratoconus.

\section{Materials and methods}

The eyes of patients with a diagnosis of progressive keratoconus who had C-CXL ( $3 \mathrm{~mW} / \mathrm{cm}^{2}$ for 30 minutes) or TE-ACXL ( $6 \mathrm{~mW} / \mathrm{cm}^{2}$ for 15 minutes) between 2015 and 2017 were included in this retrospective interventional study. The medical records of all patients who underwent CXL and followed in the Ophthalmology Corneal Department of Centro Hospitalar São João were reviewed. The main inclusion criteria were age between 14 and 32 years, a corneal pachymetry $>400 \mu \mathrm{m}$ at its thinnest point, and documented keratoconus progression.

The diagnosis of progressive keratoconus was defined by at least one of the following clinical signs in the previous 12 months: an increase of at least 1.0 diopter (D) in the maximum keratometry, a $2 \%$ decrease in central thickness, or an increase in corneal cylinder of at least 1.0 D..$^{26,27}$

The exclusion criteria for CXL were apical corneal scarring, delayed epithelial healing, severe dry eye, ocular infections, connective tissue disease, pregnancy, and lactation. ${ }^{7}$

All patients included had an ophthalmologic examination before collagen crosslinking and at 2, 6, and 12 months postoperatively. Best-corrected visual acuity (BCVA) was recorded using a Snellen chart and converted to the logarithm of minimal angle of resolution (logMAR) units for statistical analysis. ${ }^{28}$ Maximum keratometry (Kmax), mean keratometry (Kmean), pachymetry of the thinnest location, index of surface variance (ISV), index of vertical asymmetry (IVA), and index of height decentration (IHD) were recorded using Pentacam (Pentacam HR; Oculus Optikgeräte GmbH, Wetzlar, Germany) before surgery and at all time points.

The tenets of the Declaration of Helsinki were followed and the approval of the Ethics Committee of Centro Hospitalar São João was obtained. Patient consent to review their medical records was not required, given that patient data confidentiality was assured.

\section{Surgical technique}

Corneal CXL was performed under sterile conditions. About $4 \mathrm{mg} / \mathrm{mL}$ of oxybuprocaine hydrochloride eyedrops was applied on the ocular surface for topical anesthesia in both CXL protocols.

Table 1 allows the comparison of TE-ACXL and C-CXL procedures.

C-CXL was carried out with the following protocol: epithelium was loosened using a smooth spatula after being soaked for 30 seconds with a $20 \%$ alcohol solution. After epithelial debridement, $0.1 \%$ riboflavin preparation was instilled into the cornea every 2 minutes for 30 minutes, and then anterior chamber flare was checked to ensure the saturation of the corneal stroma. After that the cornea was exposed to UVA light at $370 \mathrm{~nm}\left(3 \mathrm{~mW} / \mathrm{cm}^{2}\right)$ for 30 minutes. During this period, riboflavin solution was administered every 5 minutes and sterile balance sodium solution every 2 minutes to preclude excessive corneal dehydration. After the procedure, a bandage soft contact lens was used for 7 days. ${ }^{9}$ 
Table I Collagen crosslinking procedures

\begin{tabular}{|l|l|l|}
\hline Parameter & Transepithelial accelerated CXL & Conventional CXL \\
\hline Epithelium removal & No & Yes \\
\hline Riboflavin & $\begin{array}{l}\text { TE } 0.25 \% \text { riboflavin with BAC, EDTA, Tris, } \\
\text { and } 0.45 \% \text { phosphate buffer saline }\end{array}$ & $\begin{array}{l}\text { Isotonic } 0.1 \% \text { riboflavin with } 20.0 \% \text { dextran } \\
\text { T500, } \mathrm{Na}_{2} \mathrm{HPO}_{4}, \text { and } \mathrm{NaH}_{2} \mathrm{PO}_{4}\end{array}$ \\
\hline Soak time (minutes) & 20 & 30 \\
\hline UVA irradiation $\left(\mathrm{mW} / \mathrm{cm}^{2}\right)$ & 6 & 3 \\
\hline Irradiation time (minutes) & 15 & 30 \\
\hline Total dose intensity $\left(\mathrm{J} / \mathrm{cm}^{2}\right)$ & 5.4 & 5.4 \\
\hline
\end{tabular}

Abbreviations: CXL, crosslinking; UVA, ultraviolet A; TE, transepithelial; BAC, benzalkonium chloride; EDTA, ethylenedinaminetetraacetic acid.

TE-ACXL was carried out with the following protocol: through intact epithelium, $0.25 \%$ TE riboflavin preparation was instilled to the cornea every 2 minutes for 20 minutes, and then anterior chamber flare was checked to ensure the saturation of the corneal stroma. After that, cornea was exposed to the UVA light $\left(6 \mathrm{~mW} / \mathrm{cm}^{2}\right)$ for 15 minutes. During this period, riboflavin solution was administered every 5 minutes and sterile balance sodium solution every 2 minutes to preclude excessive corneal dehydration. ${ }^{19}$

In both groups, $0.3 \%$ ofloxacin, $0.1 \%$ dexamethasone phosphate, and $0.2 \%$ sodium hyaluronate were administered topically postoperatively.

\section{Statistical analysis}

Results are expressed as the mean \pm SD. For comparison of preoperative variables and postoperative variables, the paired $t$-test was used. Within the group, multiple-related samples were compared with Friedman ANOVA test. Differences between the two groups were tested with an independentsamples $t$-test. $P<0.05$ was considered statistically significant.
Statistical analysis was performed using IBM Statistical Package for the Social Sciences (SPSS) Statistics v25.0.

\section{Results}

The study enrolled 26 eyes of 24 patients; 16 eyes had TEACXL and 10 eyes had C-CXL. There was no statistically significant baseline difference between the groups with respect to age, preoperative BCVA, keratometry values, corneal thickness, and topometric indices (Table 2).

In all patients reviewed, there were no complications during the follow-up time.

\section{Visual acuity}

Figure 1 shows the mean BCVA over time. The withingroup analysis showed no statistically significant change in BCVA through follow-up in the TE-ACXL $(P=0.46)$ and C-CXL $(P=0.72)$ groups. No significant visual gain or loss was observed in either group.

In the TE-ACXL group, the mean variation of BCVA was $-0.07 \pm 0.18 \log$ MAR $(P=0.24)$ and $-0.04 \pm 0.18 \log$ MAR

Table 2 Baseline demographic and clinical characteristics of patients undergoing transepithelial accelerated crosslinking and conventional crosslinking

\begin{tabular}{|l|l|l|l|l|}
\hline Parameter & $\begin{array}{l}\text { Transepithelial } \\
\text { accelerated CXL }\end{array}$ & Conventional CXL & $95 \%$ Cl & P-value \\
\hline Age (years) & $18.69 \pm 5.47$ & $21.90 \pm 5.72$ & $-0.56-6.98$ & 0.09 \\
\hline Sex (male:female) & $8: 1$ & $13: 2$ & $-0.30-0.25$ & 0.85 \\
\hline BCVA (LogMAR) & $0.43 \pm 0.30$ & $0.28 \pm 0.15$ & $-0.37-0.65$ & 0.16 \\
\hline Kmax (D) & $60.80 \pm 8.17$ & $57.89 \pm 3.97$ & $-8.65-2.83$ & 0.31 \\
\hline Kmean (D) & $49.36 \pm 4.53$ & $48.92 \pm 2.82$ & $-3.75-2.87$ & 0.79 \\
\hline Pachymetria $(\mu \mathrm{m})$ & $463.13 \pm 33.68$ & $467.20 \pm 25.49$ & $-21.61-29.76$ & 0.45 \\
\hline ISV & $112.63 \pm 34.20$ & $103.30 \pm 20.34$ & $-34.09-15.44$ & 0.65 \\
\hline IVA & $1.20 \pm 0.40$ & $1.14 \pm 0.24$ & $-0.36-0.23$ & 0.65 \\
\hline IHD & $0.18 \pm 0.07$ & $0.16 \pm 0.03$ & $-0.07-0.02$ & 0.27 \\
\hline
\end{tabular}

Abbreviations: BCVA, best-corrected visual acuity; CXL, crosslinking; IHD, index of height decentration; ISV, index of surface variance; IVA, index of vertical asymmetry; Kmax, maximum keratometry; Kmean, mean keratometry; LogMAR, logarithm of minimal angle of resolution. 


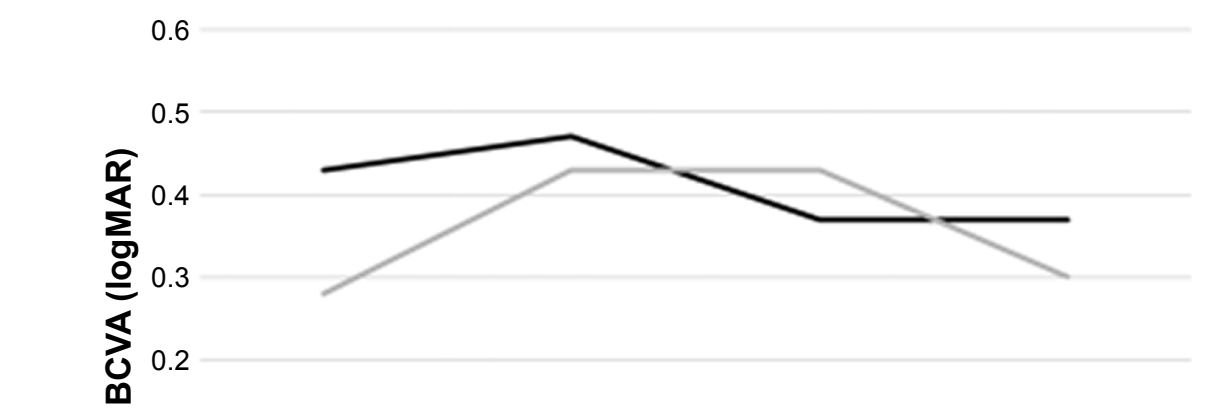

0.1

$\begin{array}{llllll} & 0 & & & & \\ & \text { Preop } & 2 \mathrm{M} & 6 \mathrm{M} & 12 \mathrm{M} & P \text {-value } \\ - \text { TE-ACXL } & 0.43 \pm 0.30 & 0.47 \pm 0.26 & 0.37 \pm 0.27 & 0.37 \pm 0.27 & 0.46 \\ - \text { C-CXL } & 0.28 \pm 0.15 & 0.43 \pm 0.36 & 0.43 \pm 0.31 & 0.30 \pm 0.13 & 0.72\end{array}$

Figure I Best-corrected visual acuity (BCVA) in LogMAR at baseline and 2, 6, and I2 months after transepithelial accelerated CXL and conventional CXL. Note: No statistically significant differences were observed between or within-group analysis at preoperative and 2, 6, and I2 months postoperatively. Abbreviations: $C X L$, crosslinking; LogMAR, logarithm of minimal angle of resolution; TE- $A C X L$, transepithelial accelerated corneal collagen CXL; C-CXL, conventional corneal collagen CXL; M, months; Preop, preoperative.

$(P=0.62)$ at 6 and 12 months, respectively. In CXL-C group, the mean variation of BCVA was $0.19 \pm 0.17 \log$ MAR $(P=0.42)$ and $0.02 \pm 0.17 \operatorname{logMAR}(P=0.82)$ at 6 and 12 months, respectively. Between-groups analysis showed no statistical significant differences at 12 months $(0.30 \pm 0.27 \log M A R$ vs $0.30 \pm 0.13 \log$ MAR, $P=0.57$ ) postoperatively.

\section{Keratometry}

Figures 2 and 3 show the variations in K readings. There were no significant changes in the Kmax and Kmean values in either group. In the TE-CXL group, variations in the keratometric values after 12 months were $\Delta \mathrm{Kmax}=-2.13 \pm 5.41$ and
$\Delta \mathrm{Kmean}=-0.25 \pm 2.60$. At the end of follow-up, no significant changes were found (Kmax and Kmean, $P=0.40$ and $P=0.78$ ). In the C-CXL group, the $\Delta \mathrm{Kmax}$ was $0.78 \pm 1.65$ and the $\Delta$ Kmean was $0.25 \pm 0.89$ at 12 months postoperatively. Also in this group, no significant changes in keratometric values were observed at the end of follow-up (Kmax and Kmean, $P=0.80$ and $P=0.31$ ).

The between-groups analysis showed no statistically significant difference between groups after 12 months. The Kmax was $57.78 \pm 6.69$ in the TE-ACXL group vs $58.67 \pm 4.45$ in the C-CXL group $(P=0.73$ ) and the Kmean was $48.22 \pm 4.47$ in the TE-ACXL group vs $49.17 \pm 2.75$ in the $\mathrm{C}$-CXL group $(P=0.57)$.

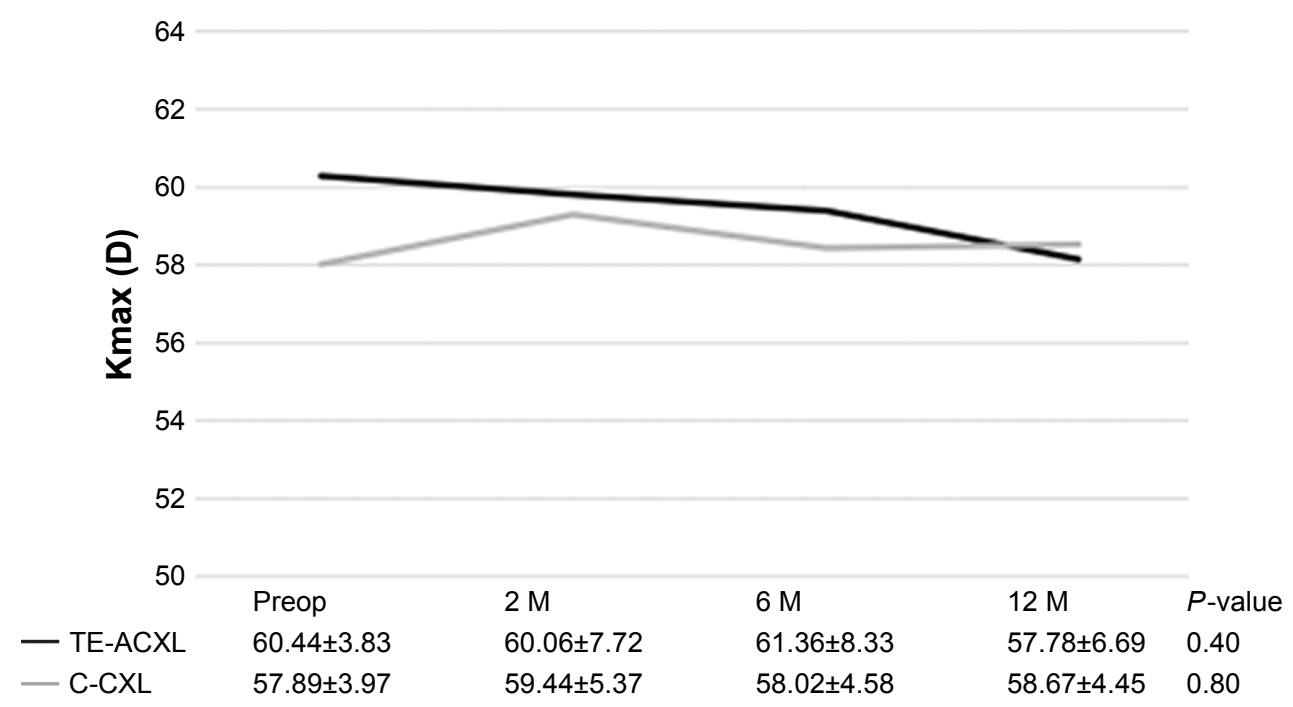

Figure 2 Maximum keratometry (Kmax) value compared with baseline at 2, 6, and 12 months after transepithelial accelerated CXL and conventional CXL. Note: No statistically significant differences were observed between or within-group analysis at preoperative and 2, 6, and I2 months postoperatively. Abbreviations: CXL, crosslinking; TE-ACXL, transepithelial accelerated corneal collagen CXL; C-CXL, conventional corneal collagen CXL; M, months; Preop, preoperative. 


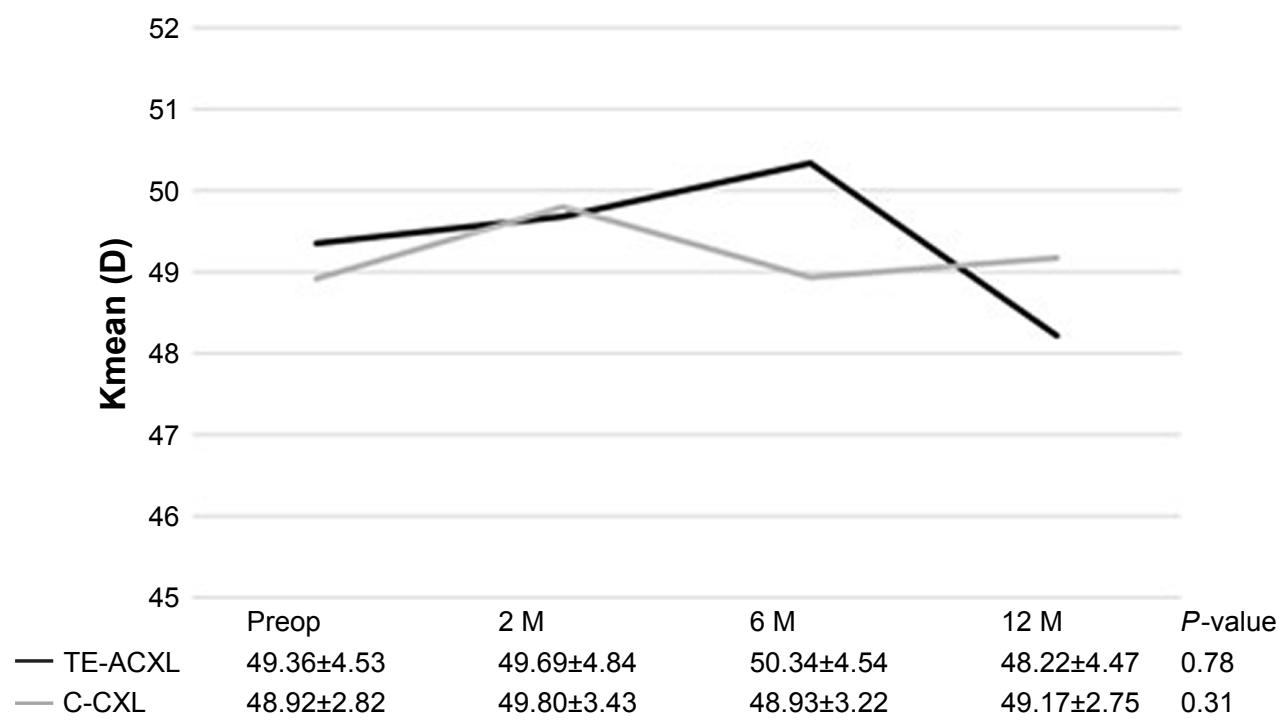

Figure $3 \mathrm{Kmean}$ values were similar at baseline, 2, 6, and 12 months postoperatively for both transepithelial accelereated CXL and conventional CXL. Note: No statistically significant differences were observed between or within-group analysis at preoperative and 2, 6, and I2 months postoperatively. Abbreviations: CXL, crosslinking; TE-ACXL, transepithelial accelerated corneal collagen CXL; C-CXL, conventional corneal collagen CXL; Kmean, mean keratometry; M, months; Preop, preoperative.

\section{Pachymetry}

Figure 4 shows the variation in pachymetry between baseline and 12 months. In both groups, no statistically significant changes were found at 12 months $(4.10 \pm 14.83, P=0.59$ in TE-ACXL group and $-8.90 \pm 22.09, P=0.42$ in C-CXL group). At the end of the follow-up, in the between-group analysis, no significant differences were observed $(458.30 \pm 24.93$ in the TE-ACXL group and $472.10 \pm 44.88$ in the $\mathrm{C}$-CXL, $P=0.37$ ).

\section{Topographic indices}

Regarding the topographic indices (Table 3), within-groups analysis showed no significant change of the variables between preoperative and 12-month postoperative readings. At 12 months, comparing the results between the TE-ACXL and the C-CXL groups, we observed that mean ISV was $104.70 \pm 37.32$ vs $107.10 \pm 22.83, P=0.86$; mean IVA was $1.12 \pm 0.39$ vs $1.17 \pm 0.31, P=0.79$; and mean IHD was $0.15 \pm 0.06$ vs $0.16 \pm 0.31, P=0.86$.

\section{Discussion}

Since the first published results in 2003 by Wollensak et al, CXL has been widely used to delay progression in patients with keratoconus. C-CXL has been shown to be safe and effective in corneal stabilization in patients with keratoconus. ${ }^{5,9,18,19}$

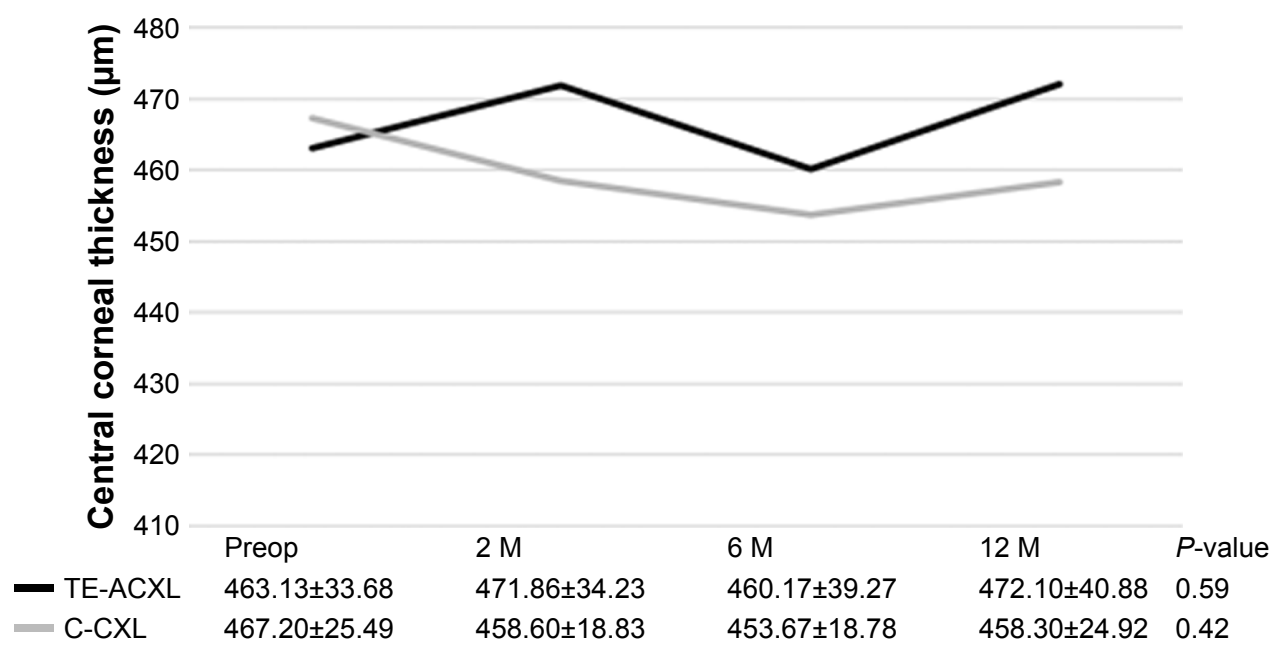

Figure 4 Central corneal thickness compared with baseline at 2, 6, and 12 months after transepithelial accelerated CXL and conventional CXL.

Note: No statistically significant differences were observed between or within-group analysis at preoperative and 2, 6, and I2 months postoperatively.

Abbreviations: CXL, crosslinking; TE-ACXL, transepithelial accelerated corneal collagen CXL; C-CXL, conventional corneal collagen CXL; M, months; Preop, preoperative. 
Table 3 Mean change in $\mathrm{K}$ readings and in topographic data in the CXL groups

\begin{tabular}{|c|c|c|c|c|c|c|}
\hline \multirow[t]{2}{*}{ Parameter } & \multicolumn{3}{|c|}{ Transepithelial accelerated CXL } & \multicolumn{3}{|c|}{ Conventional CXL } \\
\hline & $\Delta 12 M$ & $95 \% \mathrm{Cl}$ & $P$-value & $\Delta 12 M$ & $95 \% \mathrm{Cl}$ & $P$-value \\
\hline$\Delta \mathrm{Kmax}(\mathrm{D})$ & $-2 .|3 \pm 5.4|$ & $-6.00-1.74$ & 0.25 & $0.78 \pm 1.65$ & $-0.40-1.96$ & 0.17 \\
\hline$\Delta$ Kmean (D) & $-0.25 \pm 2.60$ & $-2.10-1.61$ & 0.77 & $0.25 \pm 0.89$ & $-0.39-0.89$ & 0.40 \\
\hline$\Delta$ Pachymetry $(\mu \mathrm{m})$ & $4.10 \pm 14.83$ & $-6.5|-| 4.7 \mid$ & 0.41 & $-8.90 \pm 22.09$ & $-24.70-6.90$ & 0.24 \\
\hline$\Delta \mathrm{ISV}$ & $-8.50 \pm 21.27$ & $-23.7|-6.7|$ & 0.24 & $3.80 \pm 12.43$ & $-0.5 I-12.70$ & 0.36 \\
\hline$\Delta \mathrm{IVA}$ & $-0.12 \pm 0.31$ & $-0.34-0.11$ & 0.26 & $0.03 \pm 0.18$ & $-0.10-1.62$ & 0.61 \\
\hline$\Delta \mathrm{IHD}$ & $-0.03 \pm 0.07$ & $-0.08-0.02$ & 0.18 & $-0.00 I \pm 0.03$ & $-0.02-0.02$ & 0.88 \\
\hline
\end{tabular}

Abbreviations: $\mathrm{K}$, keratometry; CXL, crosslinking; $\mathrm{M}$, months; $\mathrm{Cl}$, confidence interval; Kmax, maximum keratometry; Kmean, mean keratometry; ISV, index of surface variance; IVA, index of vertical asymmetry; IHD, index of height decentration.

However, the long duration of procedure and the need to debride the corneal epithelium lead to patient discomfort and increase in the risk of corneal infection. ${ }^{22}$ Therefore, to overcome these disadvantages, modifications and optimizations of this technique were done. In this study, we compared the safety and efficacy of TE-ACXL with C-CXL.

In our study, both TE-ACXL and C-CXL were similarly effective. Overall, patients in both groups did not show progression in clinical and topographic parameters. However, there was a trend in all the evaluated variables, with a more favorable outcome in the TE-ACXL group compared to C-CXL group.

Other studies have also demonstrated that transepithelial and accelerated crosslinking protocols may be as effective as conventional protocols; $;^{19,22,29-31}$ however, only a limited amount of studies have evaluated the efficacy of the TEACXL protocol. ${ }^{24,25}$ Until this moment, only few studies conducted in adults evaluated the efficacy of TE-ACXL; however, the protocol used was different. In one study, the cornea was exposed to UVA irradiance of $9 \mathrm{~mW} / \mathrm{cm}^{2}$ for 10 minutes, ${ }^{25}$ whilst in an other, an irradiance of $45 \mathrm{~mW} / \mathrm{cm}^{2}$ for a total dose of $7.2 \mathrm{~J}$ was used. ${ }^{24}$ The first study enrolled 26 eyes with progressive keratoconus, with a mean age of $24.54 \pm 5.16$ years and a significant improvement in BCVA and Kmax after 12 months of follow-up. ${ }^{25}$ However, in the second study, which included 46 eyes, no significant changes were observed regarding the BCVA and topographic indices, but a significant increase in corneal central thickness was observed. ${ }^{24}$ Both studies concluded that TE-ACXL is an effective method to decrease or stop the progression of keratoconus. ${ }^{24,25}$ Four more studies with TE-ACXL have been published; however, these studies used pulsed corneal irradiation instead of continuous irradiation, as in our study. ${ }^{32-35}$

In accordance with the results of CXL in previously published papers, in our study, BCVA remained stable during the 12 months of follow-up and the results were similar for both procedures. Akbar et al used a similar technique for TE-ACXL, and they did not show any significant improvement in BCVA. The BCVA was $0.43 \pm 0.30$ and $0.37 \pm 0.27$ at baseline and after 12 months, respectively $(P=0.621){ }^{25}$ However, other authors have reported BCVA improvement with transepithelial $\mathrm{CXL}^{3,23}$ and with accelerated $\mathrm{CXL} .{ }^{36}$ No articles with worsening of BCVA after transepithelial or accelerated CXL were found.

Regarding the keratometry values, the corneal flatting in the TE-ACXL group was $-2.13 \mathrm{D}$ after 12 months of follow-up; however, the difference was not statistically significant. Several studies suggested that a greater flattening effect could be achieved with more severe keratoconus patients. ${ }^{30,37}$ Heikal et al reported significant improvement in Kmax in patients who underwent transepithelial CXL (about $76.66 \%$ had a decrease by $1 \mathrm{D}$ or more), ${ }^{3}$ and Toker et al reported a statistically significant improvement in Kmax in the accelerated and C-CXL without statistical difference between then. ${ }^{38}$ However, other studies showed the opposite results. Li et al suggested that transepithelial CXL effect may not treat deeper corneal tissue because in the C-CXL group, there was a greater effectiveness in the Kmax reduction. ${ }^{39}$ Interestingly, Soeters et al have reported that in $23 \%$ of cases treated by transepithelial CXL, keratoconus progression occured. ${ }^{23}$ The differences between these studies may be related to topographic differences among the populations evaluated or differences in CXL protocols (UVA irradiation time and the riboflavin preparations used). ${ }^{30}$

Reduction in the corneal thickness has been associated with worsening of the keratoconus. In our C-CXL group, there was a decrease in corneal thickness in the first 6 months. Our results are in agreement with the published literature, since after CXL there was a significant worsening in the first 3 months with recovery beginning between 3 and 6 months. ${ }^{16,20}$ The mean change of corneal thickness in our C-CXL group may be related to epithelial remodeling, compactness of collagen 
fibrils, corneal dehydration, and keratocyte apoptosis. ${ }^{20,38}$ Compared to the C-CXL group, in the TE-ACXL group, there was a slight increase in corneal thickness at 12 months, which suggests an effective structural effect. ${ }^{38}$

Of all the topographic indices, ISV and IHD have a better correlation with the progression of keratoconus. ${ }^{27}$ In our study, there were no statistically significant differences between the ISV, IVA, and IHD indices when both treatment groups were compared. Toker et al and Choi et al reported similar results. ${ }^{15,20}$

The strength of our study is that given the lack of data on the safety and efficacy of TE-ACXL, we provided unique data on that topic. ${ }^{38,39}$ Our results prove that TE-ACXL is effective in stabilizing the progression of keratoconus. TE-ACXL is performed without epithelium debridement, thereby decreasing the risk of corneal infection, haze, and postoperative pain. ${ }^{31,40}$ Also this protocol is less time-consuming than the conventional one and facilitates the use of CXL for less co-operative patients. ${ }^{3}$ The advantages of this protocol over the conventional one make it a more appealing option for keratoconus treatment.

However, several limitations should be considered in this study. First, our sample size was small, which reduced the statistical power of the study. Second, the follow-up time was short. The progression of keratoconus was not linear over time, and there were moments when progression interspersed with periods of stabilization. Thus, follow-up of 12 months was not enough to classify assertively the keratoconus as stable, and it is believed that this flatting process induced by crosslinking can continue for several years. ${ }^{23}$

\section{Conclusion}

TE-ACXL and C-CXL seem to be similarly effective. TEACXL is a less invasive and faster procedure that seems to be effective in halting keratoconus progression. Further studies with longer follow-up and larger sample sizes are required to achieve meaningful conclusions and allow the predictability of the outcome.

\section{Disclosure}

The authors report no conflicts of interest in this work.

\section{References}

1. Mas Tur V, Macgregor C, Jayaswal R, O’Brart D, Maycock N. A review of keratoconus: diagnosis, pathophysiology, and genetics. Surv Ophthalmol. 2017;62(6):770-783.

2. Kobashi H, Rong SS. Corneal collagen cross-linking for keratoconus: systematic review. Biomed Res Int. 2017;2017:8145651.

3. Heikal MA, Soliman TT, Fayed A, Hamed AM. Efficacy of transepithelial corneal collagen crosslinking for keratoconus: 12-month follow-up. Clin Ophthalmol. 2017;11:767-771.
4. Godefrooij DA, de Wit GA, Uiterwaal CS, Imhof SM, Wisse RP Age-specific incidence and prevalence of keratoconus: a nationwide registration study. Am J Ophthalmol. 2017;175:169-172.

5. Tomita M, Mita M, Huseynova T. Accelerated versus conventional corneal collagen crosslinking. J Cataract Refract Surg. 2014;40(6): 1013-1020.

6. Alió JL, Vega-Estrada A, Sanz-Díez P, Peña-García P, DuránGarcía ML, Maldonado M. Keratoconus management guidelines. Int J Keratoconus Ectatic Corneal Dis. 2015;4(April):1-39.

7. Gomes JAP, Rapuano CJ, Belin MW, Ambrósio R. Group of panelists for the global delphi panel of keratoconus and ectatic diseases. Reply to "Global consensus on keratoconus and ectatic diseases". Cornea. 2015;34(9):e27-e29.

8. Andreanos KD, Hashemi K, Petrelli M, Droutsas K, Georgalas I, Kymionis GD. Keratoconus treatment algorithm. Ophthalmol Ther. 2017;6(2):245-262.

9. Wollensak G, Spoerl E, Seiler T. Riboflavin/ultraviolet-a-induced collagen crosslinking for the treatment of keratoconus. Am J Ophthalmol. 2003;135(5):620-627.

10. Hagem AM, Thorsrud A, Sandvik GF, Råen M, Drolsum L. Collagen crosslinking with conventional and accelerated ultraviolet-A irradiation using riboflavin with hydroxypropyl methylcellulose. J Cataract Refract Surg. 2017;43(4):511-517.

11. Konstantopoulos A, Mehta JS. Conventional versus accelerated collagen cross-linking for keratoconus. Eye Contact Lens. 2015;41(2):65-71.

12. Wittig-Silva C, Chan E, Islam FM, Wu T, Whiting M, Snibson GR. A randomized, controlled trial of corneal collagen cross-linking in progressive keratoconus: three-year results. Ophthalmology. 2014;121(4): 812-821.

13. Hashemi H, Seyedian MA, Miraftab M, Fotouhi A, Asgari S. Corneal collagen cross-linking with riboflavin and ultraviolet A irradiation for keratoconus: long-term results. Ophthalmology. 2013;120(8): $1515-1520$

14. Raiskup-Wolf F, Hoyer A, Spoerl E, Pillunat LE. Collagen crosslinking with riboflavin and ultraviolet-A light in keratoconus: long-term results. J Cataract Refract Surg. 2008;34(5):796-801.

15. Toker E, Çerman E, Özcan DÖ, Seferoğlu ÖB. Efficacy of different accelerated corneal crosslinking protocols for progressive keratoconus. J Cataract Refract Surg. 2017;43(8):1089-1099.

16. Chang CY, Hersh PS. Corneal collagen cross-linking. Eye Contact Lens. 2014;40(6):345-352.

17. Mukhtar S, Ambati BK. Pediatric keratoconus: a review of the literature. Int Ophthalmol. 2018;38(5):2257-2266.

18. Rao SK. Symposium: Keratoconus Collagen cross linking: current perspectives collagen cross linking in post LASIK. Indian J Ophthalmol. 2013;61(8):420-421.

19. Medeiros CS, Giacomin NT, Bueno RL, Ghanem RC, Moraes HV, Santhiago MR. Accelerated corneal collagen crosslinking: technique, efficacy, safety, and applications. J Cataract Refract Surg. 2016; 42(12):1826-1835.

20. Choi M, Kim J, Kim EK, Seo KY, Kim TI. Comparison of the conventional dresden protocol and accelerated protocol with higher ultraviolet intensity in corneal collagen cross-linking for keratoconus. Cornea. 2017;36(5):523-529.

21. Woo JH, Iyer JV, Lim L, et al. Conventional versus accelerated collagen cross-linking for keratoconus: a comparison of visual, refractive, topographic and biomechanical outcomes. Open Ophthalmol J. 2017;11(1):262-272.

22. O'Brart DPS. Corneal collagen crosslinking for corneal ectasias: a review. Eur J Ophthalmol. 2017;27(3):253-269.

23. Soeters N, Wisse RP, Godefrooij DA, Imhof SM, Tahzib NG. Transepithelial versus epithelium-off corneal cross-linking for the treatment of progressive keratoconus: a randomized controlled trial. Am J Ophthalmol. 2015;159(5):821-828.

24. Kır MB, Türky1lmaz K, Öner V. Transepithelial high-intensity crosslinking for the treatment of progressive keratoconus: 2-year outcomes. Curr Eye Res. 2017;42(1):28-31. 
25. Akbar B, Intisar-Ul-Haq R, Ishaq M, Arzoo S, Siddique K. Transepithelial corneal crosslinking in treatment of progressive keratoconus: 12 months' clinical results. Pak J Med Sci. 2017;33(3):570-575.

26. Shajari M, Steinwender G, Herrmann K, et al. Evaluation of keratoconus progression. Br J Ophthalmol. 2018. Epub 2018 Jun 1.

27. Kanellopoulos AJ, Asimellis G. Revisiting keratoconus diagnosis and progression classification based on evaluation of corneal asymmetry indices, derived from scheimpflug imaging in keratoconic and suspect cases. Clin Ophthalmol. 2013;7:1539-1548.

28. Holloday JT. Visual acuity measurements. J Cataract Refract Surg. 2004;30:287-290.

29. Mastropasqua L. Collagen cross-linking: when and how? A review of the state of the art of the technique and new perspectives. Eye Vis. 2015; 2(1):19.

30. Ng AL, Chan TC, Cheng AC, Alk NG, Tc C. Conventional versus accelerated corneal collagen cross-linking in the treatment of keratoconus. Clin Exp Ophthalmol. 2016;44(1):8-14.

31. Shalchi Z, Wang X, Nanavaty MA. Safety and efficacy of epithelium removal and transepithelial corneal collagen crosslinking for keratoconus. Eye. 2015;29(1):15-29.

32. Sun L, Li M, Zhang X, et al. Transepithelial accelerated corneal collagen cross-linking with higher oxygen availability for keratoconus: 1-year results. Int Ophthalmol. 2018. Epub 2017 Nov 7.

33. Zhang X, Sun L, Chen Y, Li M, Tian M, Zhou X. One-year outcomes of pachymetry and epithelium thicknesses after accelerated $(45 \mathrm{~mW} / \mathrm{cm} 2)$ transepithelial corneal collagen cross-linking for keratoconus patients. Sci Rep. 2016;6(1):1-9.
34. Artola AaRuiz-Fortes P, Soto-Negro R. Clinical outcomes at one year following keratoconus treatment with accelerated transepithelial crosslinking. Int J Ophthalmol. 2017;10(4):3-6.

35. Aixinjueluo W, Usui T, Miyai T, Toyono T, Sakisaka T, Yamagami S. Accelerated transepithelial corneal cross-linking for progressive keratoconus: a prospective study of 12 months. Br J Ophthalmol. 2017;101(9): 1244-1249.

36. Kanellopoulos AJ. Long term results of a prospective randomized bilateral eye comparison trial of higher fluence, shorter duration ultraviolet A radiation, and riboflavin collagen cross linking for progressive keratoconus. Clin Ophthalmol. 2012;6(1):97-101.

37. Greenstein SA, Hersh PS. Characteristics influencing outcomes of corneal collagen crosslinking for keratoconus and ectasia: implications for patient selection. J Cataract Refract Surg. 2013;39(8):1133-1140.

38. Çerman E, Toker E, Ozarslan Ozcan D. Transepithelial versus epithelium-off crosslinking in adults with progressive keratoconus. $J$ Cataract Refract Surg. 2015;41(7):1416-1425.

39. Li W, Wang B. Efficacy and safety of transepithelial corneal collagen crosslinking surgery versus standard corneal collagen crosslinking surgery for keratoconus: a meta-analysis of randomized controlled trials. BMC Ophthalmol. 2017;17(1):1-7.

40. Caporossi A, Mazzotta C, Paradiso AL, Baiocchi S, Marigliani D, Caporossi T. Transepithelial corneal collagen crosslinking for progressive keratoconus: 24-month clinical results. J Cataract Refract Surg. 2013;39(8):1157-1163.
Clinical Ophthalmology

\section{Publish your work in this journal}

Clinical Ophthalmology is an international, peer-reviewed journal covering all subspecialties within ophthalmology. Key topics include: Optometry; Visual science; Pharmacology and drug therapy in eye diseases; Basic Sciences; Primary and Secondary eye care; Patient Safety and Quality of Care Improvements. This journal is indexed on

\footnotetext{
Submit your manuscript here: http://www.dovepress.com/clinical-ophthalmology-journal
}

\section{Dovepress}

PubMed Central and CAS, and is the official journal of The Society of Clinical Ophthalmology (SCO). The manuscript management system is completely online and includes a very quick and fair peer-review system, which is all easy to use. Visit http://www.dovepress.com/ testimonials.php to read real quotes from published authors. 